\title{
Imaging of acoustic charge transport in semiconductor heterostructures by surface acoustic waves
}

Cite as: Appl. Phys. Lett. 75, 4139 (1999); https://doi.org/10.1063/1.125562

Submitted: 10 August 1999 - Accepted: 30 October 1999 • Published Online: 23 December 1999

M. Streibl, A. Wixforth, J. P. Kotthaus, et al.

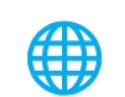

\section{ARTICLES YOU MAY BE INTERESTED IN}

Macroscopic acoustoelectric charge transport in graphene

Applied Physics Letters 103, 133101 (2013); https://doi.org/10.1063/1.4822121

Acoustic charge transport induced by the surface acoustic wave in chemical doped graphene Applied Physics Letters 109, 183110 (2016); https://doi.org/10.1063/1.4967192

Acoustically induced current flow in graphene

Applied Physics Letters 100, 133105 (2012); https://doi.org/10.1063/1.3697403

母QBLOX

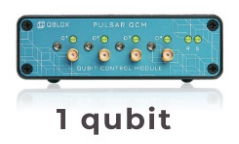

Shorten Setup Time Auto-Calibration More Qubits

Fully-integrated Quantum Control Stacks Ultrastable DC to $18.5 \mathrm{GHz}$ Synchronized $<<1$ ns Ultralow noise

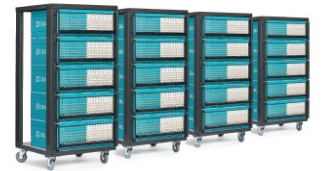

100s qubits

visit our website > 


\title{
Imaging of acoustic charge transport in semiconductor heterostructures by surface acoustic waves
}

\author{
M. Streibl, ${ }^{\text {a) }}$ A. Wixforth, and J. P. Kotthaus \\ Sektion Physik der LMU, Geschwister-Scholl-Platz 1, D-80539 München, Germany
}

\author{
A. O. Govorov \\ Institute of Semiconductor Physics, Russian Academy of Sciences, Siberian Branch, 630090 Novosibirsk, \\ Russia \\ C. Kadow and A. C. Gossard \\ Materials Department, University of California, Santa Barbara, California 93106
}

(Received 10 August 1999; accepted for publication 30 October 1999)

\begin{abstract}
We demonstrate room-temperature acoustic charge transport of electrons and holes in an InGaAs/ GaAs heterostructure. The carriers are optically generated by interband absorption and then separated, stored, and transported in the piezoelectric potential superlattice of a surface acoustic wave. The charge distribution is detected with a spatial resolution of a few acoustic wavelengths by a second orthogonal probe beam, genererated by so-called tapered transducers. The image information is given as a phase shift signal in frequency space and allows for the direct comparison of the number of generated and transported carriers. Regions of mere carrier drag and full carrier capture and transport are observed simultaneously. (c) 1999 American Institute of Physics.
\end{abstract}

[S0003-6951(99)01252-8]

Devices exploiting the electromechanical properties of surface acoustic waves (SAWs) in piezoelectric solids are state of the art signal processing tools. Because of their structural simplicity and robustness they made their way into daily life electronics. On semiconductor materials SAWs can be used to modulate the optical and electronical properties by means of the associated lattice deformation and the respective piezoelectric fields. Photogenerated electrons and holes for example can be captured in the SAWs moving potential superlattice. They can be stored and transported and their decay as a time delayed light signal can be intentially induced at the end of the SAWs path. ${ }^{1}$ At low amplitudes SAWs serve as a noninvasive detection tool for probing the properties of electron systems on an otherwise unstructured semiconductor heterostructure. They have been extensively employed in the investigation of the quantum hall regime ${ }^{2}$ and the fractional quantum hall regime ${ }^{3}$ and contributed significantly to the verification of the composite fermion picture.

In the present letter we make a two fold use of SAWs on an InGaAs/GaAs quantum well system: A high power SAW beam is employed to pump optically generated carriers from their origin in a laser spot through the sample. On their way in the acoustic path the transported carriers are observed by a second orthogonal probe SAW beam. This second probe path with a special layout not only allows for the direct measurement of the number of transported carriers with high sensitivity even at room temperature, but provides additionally a spatial resolution of a few SAW wavelengths. The experiments were performed on an $\mathrm{InGaAs} / \mathrm{GaAs}$ double quantum well structure (well width $70 \AA$ ), embedded in an AlGaAs/ GaAs optical waveguide. The structure was chosen because

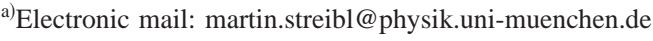

it shows good room-temperature photoluminescence (PL) efficiency and allows us to perform experiments on electrical and optical interaction mechanisms simultaneously.

A typical sample layout is shown in the inset of Fig. 1. Two orthogonal acoustic paths are defined by interdigital transducers (IDTs) for launching and detecting surface acoustic waves. The transducers are fabricated by electronbeam lithography. They consist of 100 finger pairs each with a period of $4 \mu \mathrm{m}$ in the horizontal SAW path and $3.2 \mu \mathrm{m}$ in the vertical SAW path corresponding to SAW frequencies of 740 and $980 \mathrm{MHz}$, respectively. The horizontal SAW beam is generated and detected by a network analyzer at an output power of $+5 \mathrm{dBm}$. Typical radio frequency ( $\mathrm{rf}$ ) powers for the vertical SAW beam were about $+20 \mathrm{dBm}$ with transmission losses of $-20 \mathrm{~dB}$ so $10 \%$ of the rf power is converted into acoustic power. The transducers active apertures were $400 \mu \mathrm{m}$ (IDT1 and IDT2) and $300 \mu \mathrm{m}$ (IDT3 and IDT4), respectively.

The rf transmission of the so-called tapered

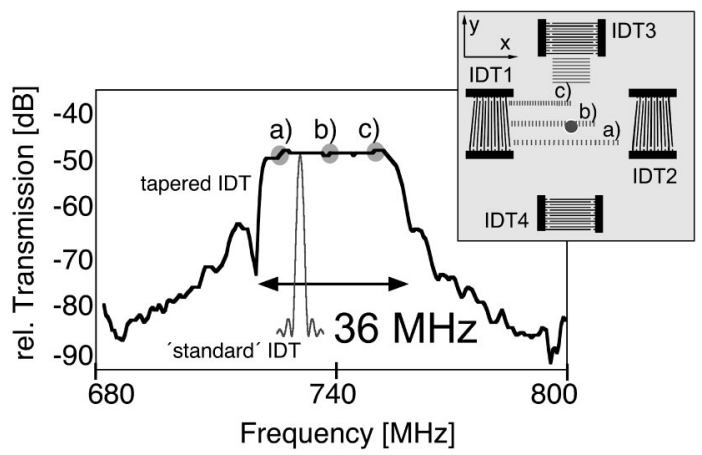

FIG. 1. Frequency response of a tapered IDT. The variation of the finger period over the transducer aperture spreads the IDT frequency response to a bandpass of $36 \mathrm{MHz}$ width. For comparison, the transmission of an untapered IDT is also shown. A schematic sample layout is sketched in the inset. 
transducers, ${ }^{4,5}$ used for exciting the probe SAW path is shown in Fig.1. The finger period in this special transducer layout changes linearly over the transducer aperture, in our case by $5 \%$. On the one hand, this results in a transmission bandpass of about $36 \mathrm{MHz}$ width as indicated in Fig. 1. On the other hand, for a fixed frequency $f$ in the bandpass an acoustic wave is only launched in a small stripe of the IDT where the resonance condition $f d(y)=\mathrm{v}_{\mathrm{SAW}}$ is matched $[d(y)$ here denotes the IDT periodicity versus coordinate $y]$. The tapered IDTs, hence, allow for the excitation of an extremely narrow SAW beam and an adjustability of the lateral beam position within the $400 \mu \mathrm{m}$ transducer aperture. Because of the linear relation between frequency and the lateral position of the SAW beam we get a translation factor from frequency space to real space of $11 \mu \mathrm{m} / \mathrm{MHz}$. From the typical bandwidth of a conventional untapered IDT of less than 2 $\mathrm{MHz}$ we estimate a width of the acoustic path below $20 \mu \mathrm{m}$. Recent $x$-ray topography experiments at the ESRF in Grenoble $^{6}$ allow for the direct observation of the SAW beam and confirm this estimate. Of course, as the width of the acoustic path is of the order of the acoustic wavelength diffraction effects are to be expected. In fact, we find a $10 \mathrm{~dB}$ decrease of the SAW transmission when the degree of tapering is increased from $5 \%$ to $10 \%$ which clearly stresses the importance of diffraction. However, as the second receiving transducer has the same selectivity, only the undisturbed components of the wave are detected keeping the relevant beam width unaffected from diffraction effects.

In our experiments we use a TiSa laser beam $(\lambda$ $=860 \mathrm{~nm})$ at typical intensities from 10 to $850 \mu \mathrm{W}$ continuous wave and focused down to micrometer spotsize by a microscope objective to generate an electron hole plasma (EHP) in the SAW path. The acoustoelectic interaction between free carriers and the SAW probe beam can be used to scan and image the photogenerated charge distribution: SAWs in GaAs couple to a conductive sheet near the surface by means of their accompanying piezoelectric fields. Some of the waves energy is dissipated within the electron/hole system by screening currents. The interaction of electronhole $(e-h)$ pairs and the SAW results in a velocity change of the wave and a corresponding phase shift. The change in acoustic velocity is given by ${ }^{7}$

$$
\frac{\Delta \mathrm{v}}{\mathrm{V}}=\frac{k_{\mathrm{eff}}^{2}}{2} \frac{1}{1+\left(\sigma / \sigma_{M}\right)^{2}}
$$

where $k_{\text {eff }}^{2}=6.4 \times 10^{-4}$ is the electromechanical coupling constant for GaAs, $\sigma$ is the conductivity of a conducting sheet near the surface and $\sigma_{M} \approx 3 \times 10^{-7} \Omega_{\square}^{-1}$ is the critical conductivity for GaAs where energy transfer from a SAW into this sheet is maximum. The charge distribution in the laser spot is well approximated by a gaussian distribution ${ }^{8}$ which motivates the assumption of a gaussian conductivity profile $\sigma(x, y)=\sigma_{0} \sigma_{M} \exp \left(-\left(x^{2}+y^{2}\right) / w^{2}\right)$. The constant $\sigma_{0}$ here gives the maximum conductivity in units of $\sigma_{M}$. The phase shift of a SAW propagating along the $x$ direction and crossing this conductive area at position $y$ is calculated to be

$$
\Delta \phi=\frac{\pi k_{\mathrm{eff}}^{2}}{\lambda_{\mathrm{SAW}}} \int_{0}^{L} \frac{1}{1+\left[\sigma(x, y) / \sigma_{M}\right]^{2}} d x,
$$

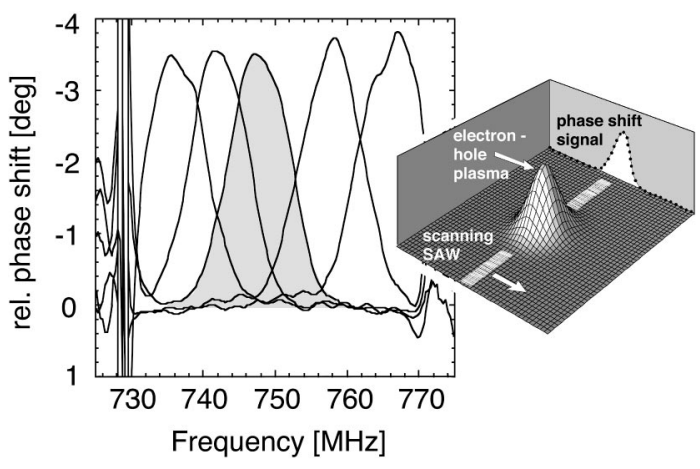

FIG. 2. Images of a photogenerated electron hole plasma at different laser spot positions within the tapered IDTs "field of view." The hatched trace corresponds to the fitting parameters given in the text. The inset schematically explains the origin of the phase signal.

where $L$ corresponds to the length of the acoustic probe path. Exciting a SAW pulse at the tapered transducer IDT1 and evaluating the phase shift of the detected rf signal at IDT2 vs SAW frequency gives a one-dimensional image of the carrier density within the acoustic path. In the measurement of Fig. 2 the laser spot was shifted through the horizontal SAW path and for each spot position the carrier distribution was scanned by the adjustable SAW beam as indicated in the inset of Fig. 2. The traces show the profile of the laser genererated electron hole plasma moving through the field of view of the probe beam. Numerical fitting of Eq. (2) reproduces the shape of the laser spots image well, but underestimates the amplitude of phase change by a factor of two. This could be explained by assuming some of the carriers are dragged along with the probe SAW pulse which elongates the profile along the direction of propagation. This intensifies the interaction between the carriers and the SAW. Accepting this factor of two, we get from the fit for the experimental situation in Fig. $2 w=66 \mu \mathrm{m}$ and $\sigma_{0}=1.46$. The laser spot image is obviously much larger than the diameter of the laser spot on the sample suggesting unusually large diffusion lengths. The large diameter can not be explained by the finite width of the scanning acoustic beam which in fact has been included in the numerical fitting procedure. Moreover, the extent of the electron-hole plasma is predominantly given by the maximum phase shift of $3.5^{\circ}$ in Fig. 2 which corresponds to a minimum interaction length of SAW and carrier system. The unusually large diameter may be explained on the one hand by the exceptionally high carrier lifetimes in our high quality samples of $\tau \geqslant 20 \mathrm{~ns}$ as determined by time resolved PL experiments. At the low amplitude of the probe SAW beam we do not expect a further increase of the lifetime by charge separation. On the other hand, the samples optical waveguide may contribute to the increase of the area of carrier generation as PL is effectively guided and reabsorbed in the vicinity of the laser spot. However, the room-temperature carrier dynamics in our structures will be further investigated in the near future.

At low temperatures acoustic phonons seem to play an important role for the expansion of an EHP. A series of publications demonstrated that the dynamics of an EHP is governed by a phonon wind mechanism. ${ }^{8,9}$ The thermalization of photoexcited carriers in the laser spot results in a so-called phonon hot spot emitting ballistic phonons. Elec- 


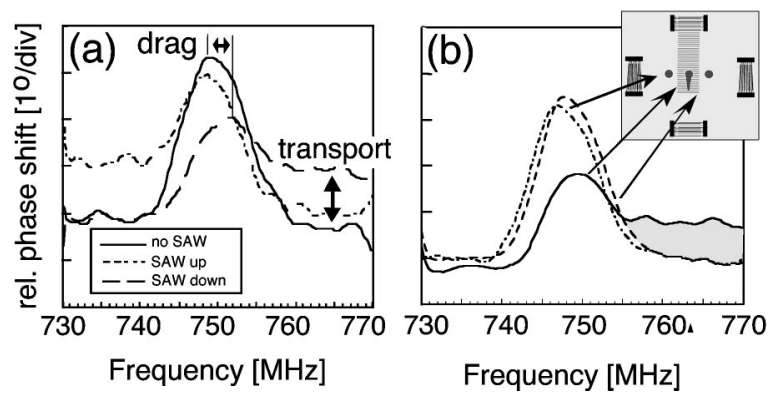

FIG. 3. (a) "Crosswind" experiment employing two orthogonal SAW beams. The swept beam (left to right) is used to monitor the photogenerated charge distribution influenced by the phonon crosswind of the second SAW (vertical path). The crosswind leads to the occurrence of a "comets tail", pointing away from the phonon source. Upon changing the direction of the cross wind SAW, this tail changes position. (b) Three images taken at various positions with respect to the crosswind beam. The comets tail only exists within this SAW beam.

trons and holes are carried away from the spot along with the phonon wind at the speed of sound. As SAWs provide an intense, coherent, and adjustable phonon wind, they are an ideal tool to study these processes in detail. In our sample the vertical pump SAW is used to generate the coherent phonon wind. The horizontal SAW beam probes the crosswind effect on the photogenerated charge distribution. In Fig. 3(a) we centered the laser spot on both the horizontal and the vertical SAW path. Without pump SAW we get a symmetric phase shift image of the laser induced charge density distribution. When the pump SAW is launched from IDT3 we observe a tail in the carrier density stretching towards IDT4 indicating acoustic charge transport. When the direction of the pump SAW is reversed the tail extends in the opposite direction. A second test is done in Fig. 3(b) where the laser spot was moved along the horizontal probe beam across the vertical pump SAW line. We again observe the occurrence of a charge density tail as soon as the spot enters the pump SAW path. Additionally to the transport tail a shift of the EHP core can be observed. In the core region dynamic screening of the SAW fields prevents a complete localization of carriers in the SAWs potential. However, momentum is still transferred to the EHP leading to the observed peak shift. The selfconsistent interplay between dynamic screening of the SAW field in regions of high EHP density and carrier drag in regions of lower density is nicely expressed by the onset of transport at the rim of the EHP. This transition from carrier drag to transport has been discussed in detail by Rotter et al. ${ }^{10}$ for a two-dimensional electron gas on a hybrid $\mathrm{GaAs} / \mathrm{LiNbO}_{3}$ system.

To study the effect of dynamic screening, we varied the intensity of the laser beam, i.e., the density of the EHP plasma. Figure 4 shows scans of the conductivity profiles of the laser spot and the acoustic transport tail for several laser intensities between $10 \mu \mathrm{W}$ and $850 \mu \mathrm{W}$. The conductivity measured in the laser spot increases almost linear with the laser intensity. Simultaneously the increasing screening effect reduces the momentum transfer on the carriers, i.e., the EHP core shift as shown in Fig. 4(b). The conductivity in the transport tail saturates at about $0.3-0.4 \sigma_{M}$ expressing the maximum transport capacity of the SAW beam. At higher densities, electrons and holes are no longer localized in the

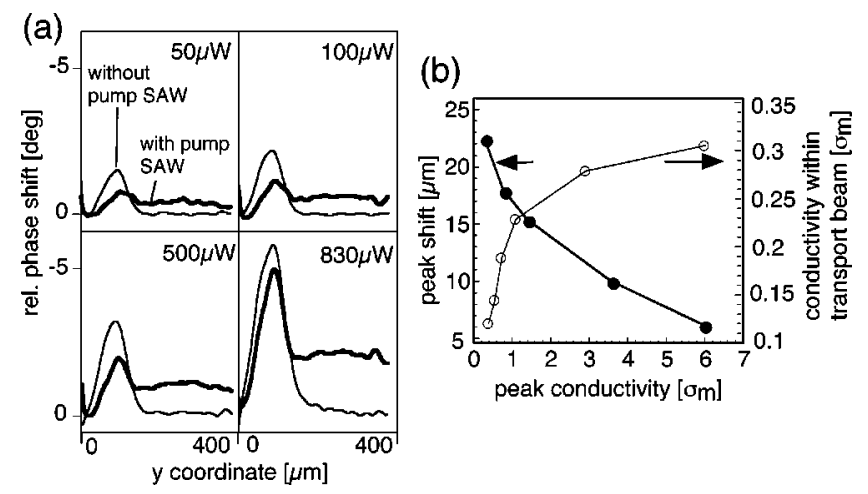

FIG. 4. (a) Phase shift image of the laser spot and the acoustic transport tail for various laser intensities. (b) Drag of the laser spot core (closed circles) and conductivity in the charge transport tail (open circles) vs conductivity in the core region. Dynamic screening at high carrier densities reduces both the drag of the EHP core and the transport efficiency. The latter is expressed by a saturation of the conductivity in the transport tail.

SAWs potential pattern and can leave the beam or are lost by recombination. Hence, the highest transport efficiency is found at the lowest laser intensities. Here the ratio of the conductivities in the transport tail and the core region reaches values up to $50 \%$. Remarkably, the transported carrier density does not drop significantly along the transport tail, which proves that carrier confinement in the SAW is high even at room temperature.

To conclude we have demonstrated electron-hole drag and transport by SAW in semiconductor heterostructures at room temperature and presented a SAW imaging technique which allows us to probe conductivity profiles with micrometer resolution. In our SAW transport experiments we were able to directly compare the carrier density in the exciting laser spot and in the carrier transport path. The experiments show that SAW driven transport of electrons and holes is efficient even at room temperature which is an important precondition for possible applications based on acoustic charge transport.

The authors gratefully acknowledge the financial support by the Bayerische Forschungsstiftung BayFor (294d/98) and the Deutsche Forschungsgemeinschaft DFG. The work at UCSB has been supported through AFOSR (Grant No. F49620-94-1-0158) and the center for quantized electronic structures (QUEST).

${ }^{1}$ C. Rocke, S. Zimmermann, A. Wixforth, J. P. Kotthaus, G. Böhm, and G. Weimann, Phys. Rev. Lett. 78, 4099 (1997).

${ }^{2}$ A. Wixforth, J. Scriba, M. Wassermeier, J. P. Kotthaus, G. Weimann, and W. Schlapp, Phys. Rev. B 40, 7874 (1989).

${ }^{3}$ R. L. Willet, R. R. Ruel, W. West, and L. N. Pfeiffer, Phys. Rev. Lett. 71, 3846 (1993).

${ }^{4}$ A. P. Van de Heuvel, Appl. Phys. Lett. 21, 280 (1972).

${ }^{5}$ L. P. Solie, IEEE Ultrason. Symp. 1986, pp. 77-80.

${ }^{6}$ W. Sauer, M. Streibl, T. H. Metzger, A. G. C. Haubrich, S. Manus, A. Wixforth, J. Peisl, A. Mazuelas, J. Härtwig, and J. Baruchel, Appl. Phys. Lett. 75, 1709 (1999).

${ }^{7}$ A. Wixforth, J. P. Kotthaus, and G. Weimann, Phys. Rev. Lett. 56, 2104 (1986).

${ }^{8}$ L. M. Smith, J. S. Preston, J. P. Wolfe, D. R. Wake, J. Klem, T. Henderson, and H. Morkoc, Phys. Rev. B 39, 1862 (1989).

${ }^{9}$ A. E. Bulatov and S. G. Tikhodeev, Phys. Rev. B 46, 15058 (1992).

${ }^{10}$ M. Rotter, A. V. Kalameitsev, A. O. Govorov, W. Ruile, and A. Wixforth, Phys. Rev. Lett. 82, 2171 (1999). 